\title{
MSP Binaries as Astrophysical Laboratories
}

\author{
Zorawar Wadiasingh ${ }^{1}$, Alice K. Harding ${ }^{2}$, Christo Venter ${ }^{1}$, \\ Markus Böttcher ${ }^{1}$ and Matthew G. Baring ${ }^{3}$ \\ ${ }^{1}$ Centre For Space Research, North-West University, Potchefstroom, South Africa \\ ${ }^{2}$ NASA Goddard Space Flight Center, Greenbelt, Maryland, USA \\ ${ }^{3}$ Rice University, Houston, Texas, USA
}

\begin{abstract}
Follow-up of unidentified Fermi sources has expanded the number of known galacticfield "black widow" and "redback" millisecond pulsar binaries from four to nearly 30. Several systems observed by Chandra, XMM-Newton, Suzaku, and NuSTAR exhibit double-peaked Xray orbital modulation. This is attributed to synchrotron emission from electrons accelerated in an intrabinary shock and Doppler boosting by mildly relativistic bulk flow. We briefly discuss the rich complexity of these systems, their astrophysical utility, and open questions.
\end{abstract}

Keywords. radiation mechanisms: nonthermal, pulsars: general, X-rays: binaries, shock waves, acceleration of particles

\section{Background}

Fermi has enabled a huge proliferation in discovered millisecond pulsars (MSPs) $\dagger$. A large fraction of these non-accreting MSPs are in binary systems with a low-mass $m_{\mathrm{c}} \ll M_{\odot}$ stellar companion in compact $<1$ day orbits corresponding to semi-major axes of $a \sim 10^{11} \mathrm{~cm} \approx 0.006 \mathrm{AU}$. These systems are colloquially known as the "arachnids" with two populations: black widows (BWs: $m_{\mathrm{c}} \lesssim 0.1 M_{\odot}$ ) and redbacks (RBs: $m_{\mathrm{c}} \gtrsim 0.2 M_{\odot}$ ) (Roberts 2011). Some RBs, e.g., J1023+0038 (Archibald et al. 2009), are "missing link" transitional MSPs that manifest epochs of accretion similar to low-mass X-ray binaries.

The multiwavelength phenomenology of these systems is rich. The radio MSP often exhibits frequency-dependent eclipses and perhaps even lensing by plasma from the irradiated companion. BWs exhibit much smaller orbital eclipse fractions and more regularity in eclipses than RBs. Due to irradiation by the MSP, the companion is much brighter than a main-sequence star of similar mass. Optical and radio mass functions, combined with models of irradiation, allow for an estimate of the MSP mass (e.g., van Kerkwijk et al. 2011). Nonthermal and double-peaked orbitally-modulated soft X-ray emission is observed in many systems. This is attributed to synchrotron emission from electrons accelerated in an intrabinary shock and Doppler boosting along the shock. The emission exhibits very hard power laws (PLs) up to $N u S T A R$ energies, with photon indices $\Gamma_{\mathrm{X}} \sim 1-1.3$, implying efficient particle acceleration.

For almost all systems, the double-peak modulation is centered around inferior pulsar conjunction. In Wadiasingh et al. (2017a) $\ddagger$ (hereafter W17a) we deduced that this is due to shock configuration bowed around the MSP. We found LC shape is a function of shock/orbital geometry and bulk flow Lorentz factor. The X-ray phasing is the relevant discriminant of the shock orientation. The PL is anticipated to extend to the MeV regime, potentially discoverable with future instruments, e.g., AMEGOף.

$\dagger$ See the Fermi Public List of LAT-Detected Gamma-Ray Pulsars

$\ddagger$ see also Romani \& Sanchez (2016)

I https://asd.gsfc.nasa.gov/amego/ 


\section{Probing Relativistic Shocks and Pulsar Winds}

Particle acceleration mechanisms are of fundamental importance in high-energy astrophysics. Kinetic simulations of shock acceleration are limited in dimensionality and cannot capture the large physical separation of scales and nonlinear plasma interactions. The LCs of BWs/RBs are potentially rich probes of shock acceleration. MSP MHD wind structure and spin alignment with the orbital axis likely results in a quasi-perpendicular shock at the nose and a quasi-parallel one in the wings. As established in W17a, the LCs are energy-dependent if the emitting PL index is spatially-dependent. This enables a fascinating empirical probe of the particle acceleration PL index distribution along a shock, and couples to theories of oblique shock acceleration.

Likewise, pulsar wind content may be probed by searches of orbitally-modulated $\gamma$ ray emission in hot BWs, as intimated in W17a. The photon field of the companion provides targets for inverse Compton scattering (ICS) by relativistic pairs in the pulsar wind. Characterization of such ICS may constrain particle anisotropy of pulsar winds. In contrast to shock emission, ICS orbital modulation flux is anticipated to be broadly singlepeaked near pulsar superior conjunction. Contrastingly, inferior conjunction phasing may be exhibited if significant particle acceleration occurs near the companion.

\section{Implications by Stability}

By energetics, a configuration where the shock enshrouds the pulsar requires that the companion wind is gravitationally captured. As we show in an upcoming paper (Wadiasingh et al. 2017b), a plausible model is an advection-dominated accretion flow (ADAF). Such capture is inherently unstable on dynamical timescales without stability mechanisms. Irradiation feedback from the shock emission on the companion is a potential stability mechanism, and implies correlated X-ray/optical variability. This scenario also limits $F_{\gamma} / F_{\mathrm{X}} \lesssim 14$ for the ratio of MSP $\gamma$-ray magnetospheric emission $F_{\gamma}$ to nonthermal shock emission $F_{\mathrm{X}}$. Such a limit, which is satisfied for $N u S T A R$ RBs, implies extension of PL shock emission to tens or hundreds of $\mathrm{keV}$ in several other RBs.

The ADAF also generates a soft X-ray/UV thermal bremsstrahlung component with cut off $\sim 3 \mathrm{keV}$. As set forth in (Wadiasingh et al. 2017b), under certain parameter choices, this component may dominate the shock synchrotron emission at orbital phases near superior conjunction and exhibit stochastic variability uncorrelated with orbital phase.

\section{Conclusions and Open Questions}

MSP binaries may serve as useful astrophysical probes for a variety of nonthermal phenomena. There are several avenues of future observational investigation. Does correlated X-ray/optical variability exist in RBs? Is there a sub-dominant UV component? What is the mechanism/nature of particle acceleration in the termination shock and what are its influences on the energy dependence of high-energy orbital modulation?

\section{References}

Archibald, A. M., Stairs, I. H., Ransom, S. M., et al. 2009, Science, 324, 1411

van Kerkwijk, M. H., Breton, R. P., \& Kulkarni, S. R. 2011, ApJ, 728, 95

Roberts, M. S. E. 2011, American Institute of Physics Conference Series, 1357, 127

Romani, R. W. \& Sanchez, N. 2016, ApJ, 828, 7

Wadiasingh, Z., Harding, A. K., Venter, C., Böttcher, M., \& Baring, M. G. 2017, ApJ, 839, 80

Wadiasingh, Z., Böttcher, M., Venter, C., \& Harding, A. K. 2017 submitted to ApJ 